\title{
Erratum to: On the Crank-Nicolson scheme once again
}

\author{
JAN A. VAN CASTEREN
}

Erratum to: J. Evol. Equ. DOI 10.1007/s00028-010-0084-9

Abstract. Let $\left(\tau_{j}\right)_{j \in \mathbb{N}}$ be a sequence of strictly positive real numbers, and let $A$ be the generator of a bounded analytic semigroup in a Banach space $X$. Put $A_{n}=\prod_{j=1}^{n}\left(I+\frac{1}{2} \tau_{j} A\right)\left(I-\frac{1}{2} \tau_{j} A\right)^{-1}$, and let $x \in X$. Define the sequence $\left(x_{n}\right)_{n \in \mathbb{N}} \subset X$ by the Crank-Nicolson scheme: $x_{n}=A_{n} x$. In this erratum, it is proved that the Crank-Nicolson scheme is stable in the sense that $\sup _{n \in \mathbb{N}}\left\|A_{n} x\right\|<\infty$ provided that inequality $(0.9)$ below holds.

Unfortunately, there is a serious flaw in the proof of Proposition 2.7, which jeopardizes the main stability result of Van Casteren [1], which is Theorem 3.1, and as a consequence, several others are affected as well: Corollaries 3.6, 4.7, and Theorems 4.3, 4.4. The author regrets this mistake. A correct formulation of Theorem 3.1 reads as follows.

THEOREM 0.1. Let $A$ be the generator of a bounded analytic semigroup, and let $\left(\tau_{j}\right)_{j \in \mathbb{N}}$ be a sequence of strictly positive numbers. Define the constant $C_{4}$ and the functions $a_{2 k}(\eta), b_{2 k}(\eta), k \in \mathbb{N}, \eta>0$, respectively, by (see equality (3.4) in the original paper [1])

$$
\begin{aligned}
& C_{4}=\sup _{\xi \in \mathbb{R}, \omega>0}\left\|\left(2 \xi^{2}\left(\xi^{2} I+(\omega I-A)^{2}\right)^{-1}+I\right)\left(\xi^{2}\left(\xi^{2} I+(\omega I-A)^{2}\right)^{-1}-I\right)\right\| \\
& a_{2 k}(\eta)=\frac{\left(2 \sum_{j=1}^{2 k} \arctan \left(\frac{1}{2} \tau_{j} \eta\right)\right) \wedge\left(2 \sum_{j=1}^{2 k} \arctan \left(\frac{2}{\tau_{j} \eta}\right)\right)}{\sum_{j=1}^{2 k} \frac{\tau_{j} \eta}{1+\frac{1}{4} \tau_{j}^{2} \eta^{2}}}, \text { and } \\
& b_{2 k}(\eta)=\frac{\sin \left(\frac{2}{2 k} \sum_{j=1}^{2 k} \arctan \left(\frac{1}{2} \tau_{j} \eta\right)\right)}{\frac{1}{2 k} \sum_{j=1}^{2 k} \frac{\tau_{j} \eta}{1+\frac{1}{4} \tau_{j}^{2} \eta^{2}}}
\end{aligned}
$$

Mathematics Subject Classification (2000): 47D06, 65J10

Keywords: Crank-Nicolson scheme, Bounded analytic semigroup.

The author is grateful to Rainer Nagel and an anonymous referee for their comments. He also thanks the University of Antwerp and the Flemish Fund for Scientific Research for their financial and logistic support.

The online version of the original article can be found under doi:10.1007/s00028-010-0084-9. 
Then $b_{2 k}(\eta) \leq a_{2 k}(\eta), \eta>0$, and for $\omega \geq 0, k \in \mathbb{N}$, the following inequalities hold:

$$
\begin{aligned}
& \left\|\prod_{j=1}^{2 k}\left(I-\frac{1}{2} \tau_{j}(\omega I-A)\right)\left(I+\frac{1}{2} \tau_{j}(\omega I-A)\right)^{-1}-I\right\| \\
& \quad \leq 4 C_{4}\left(\sup _{\eta>0}\left(b_{2 k}(\eta)\right)\right)^{2} \leq 4 C_{4}\left(\sup _{\eta>0} a_{2 k}(\eta)\right)^{2} .
\end{aligned}
$$

So in order that the Crank-Nicolson scheme with step sizes $\tau_{j}>0, j \in \mathbb{N}$, is stable, it suffices to assume that the sequence of functions $\eta \mapsto b_{2 k}(\eta), \eta>0, k \in \mathbb{N}$, is uniformly bounded. The latter is the case provided that the sequence of derivatives $\eta \mapsto b_{2 k}^{\prime}(\eta), \eta>0, k \in \mathbb{N}$, is uniformly bounded. This is a consequence of the theorem of Arzela-Ascoli together with the equalities:

$$
\lim _{\eta \downarrow 0} b_{2 k}(\eta)=\lim _{\eta \uparrow \infty} b_{2 k}(\eta)=1 .
$$

A similar remark applies to the sequence $\eta \mapsto a_{2 k}(\eta), \eta>0, k \in \mathbb{N}$. It is not clear how such conditions are reflected in the properties of the sequence $\left(\tau_{j}\right)_{j \in \mathbb{N}}$, but see Theorem 0.4 below.

Proof of Theorem 0.1 Let $\omega \geq 0$, and like in the proof of Theorem 3.1, put $\varphi_{j}(\eta)=2$ $\arctan \left(\frac{1}{2} \tau_{j} \eta\right)$ and $f_{2 k}(\eta)=2 \sum_{j=1}^{2 k} \arctan \left(\frac{1}{2} \tau_{j} \eta\right), \eta>0$. Then, a calculation gives $\eta \varphi_{j}^{\prime}(\eta)=\sin \varphi_{j}(\eta)$. As in the (wrong) proof of Theorem 3.1 in the cited paper, we have:

$$
\begin{aligned}
& \left\|\prod_{j=1}^{2 k}\left(I-\frac{1}{2} \tau_{j}(\omega I-A)\right)\left(I+\frac{1}{2} \tau_{j}(\omega I-A)\right)^{-1}-I\right\| \\
& \quad \leq \frac{4 C_{4}}{\pi} \int_{0}^{\infty} \frac{1-\cos \left(f_{2 k}(\eta)\right)}{\eta^{2}} \frac{1}{f_{2 k}^{\prime}(\eta)} d \eta
\end{aligned}
$$

The equality $b_{2 k}(\eta)=\frac{2 k \sin \frac{f_{2 k}(\eta)}{2 k}}{\sum_{j=1}^{2 k} \sin \varphi_{j}(\eta)}$ also follows, and upon writing $\psi_{2 k}(\eta)=\frac{f_{2 k}(\eta)}{2 k}$, we obtain

$$
\begin{aligned}
& \int_{0}^{\infty} \frac{1-\cos \left(f_{2 k}(\eta)\right)}{\eta^{2}} \frac{1}{f_{2 k}^{\prime}(\eta)} d \eta=\int_{0}^{\infty} \frac{1-\cos \left(f_{2 k}(\eta)\right)}{\left(\eta f_{2 k}^{\prime}(\eta)\right)^{2}} f_{2 k}^{\prime}(\eta) d \eta \\
& =\int_{0}^{\infty} \frac{1-\cos \left(f_{2 k}(\eta)\right)}{\left(\sum_{j=1}^{2 k} \sin \varphi_{j}(\eta)\right)^{2}} f_{2 k}^{\prime}(\eta) d \eta \\
& =\int_{0}^{\infty} \frac{1-\cos \left(2 k \psi_{2 k}(\eta)\right)}{2 k \sin ^{2} \psi_{2 k}(\eta)}\left(\frac{2 k \sin \psi_{2 k}(\eta)}{\sum_{j=1}^{2 k} \sin \varphi_{j}(\eta)}\right)^{2} \psi_{2 k}^{\prime}(\eta) d \eta \\
& =\int_{0}^{\infty} \frac{1-\cos \left(2 k \psi_{2 k}(\eta)\right)}{2 k \sin ^{2} \psi_{2 k}(\eta)}\left(b_{2 k}(\eta)\right)^{2} \psi_{2 k}^{\prime}(\eta) d \eta
\end{aligned}
$$




$$
\begin{aligned}
& \leq\left(\sup _{\eta>0} b_{2 k}(\eta)\right)^{2} \int_{0}^{\infty} \frac{1-\cos \left(2 k \psi_{2 k}(\eta)\right)}{2 k \sin ^{2} \psi_{2 k}(\eta)} \psi_{2 k}^{\prime}(\eta) d \eta \\
& =\left(\sup _{\eta>0} b_{2 k}(\eta)\right)^{2} \int_{0}^{\pi} \frac{1-\cos (2 k \psi)}{2 k \sin ^{2} \psi} d \psi=\pi\left(\sup _{\eta>0} b_{2 k}(\eta)\right)^{2} .
\end{aligned}
$$

In the final step in (0.3), we used integration by parts and the trigonometric identity

$$
\cot \psi \sin (2 k \psi)=1-\cos (2 k \psi)+2 \sum_{j=1}^{k} \cos (2 j \psi), \quad k \geq 1,
$$

to obtain

$$
\begin{aligned}
\int_{0}^{\pi} \frac{1-\cos (2 k \psi)}{2 k \sin ^{2} \psi} d \psi & =\int_{0}^{\pi} \cot \psi \sin (2 k \psi) d \psi \\
& =\int_{0}^{\pi}\left(1-\cos (2 k \psi)+2 \sum_{j=1}^{k} \cos (2 j \psi)\right) d \psi=\pi
\end{aligned}
$$

Insertion of (0.3) into (0.2) yields the inequality in (0.1). Since the inequality $b_{2 k}(\eta) \leq$ $a_{2 k}(\eta)$ is elementary, this completes the proof of Theorem 0.1 .

We continue with the following remark. Let the notation be as in (the proof of) Theorem 0.1 , and put

$$
c_{2 k}(\eta)=\frac{\left(\sum_{j=1}^{2 k} \varphi_{j}(\eta)\right) \wedge\left(\sum_{j=1}^{2 k}\left(\pi-\varphi_{j}(\eta)\right)\right)}{\sum_{j=1}^{2 k} \varphi_{j}(\eta) \wedge\left(\pi-\varphi_{j}(\eta)\right)}, \quad \eta>0 .
$$

Then, it is easy to see that

$$
b_{2 k}(\eta) \leq a_{2 k}(\eta) \leq \frac{\pi}{2} c_{2 k}(\eta) \leq \frac{\pi^{2}}{4} b_{2 k}(\eta), \quad \eta \geq 0 .
$$

From (0.4), it readily follows that $a_{2 k}(\eta)$ is uniformly bounded in $\eta>0$ and $k$ if and only if this is true for $b_{2 k}(\eta)$ if and only if this is true for $c_{2 k}(\eta)$. In addition, the following inequalities are not difficult to check:

$$
\begin{aligned}
& \left(\sum_{j=1}^{2 k} \varphi_{j}(\eta)\right) \wedge\left(\sum_{j=1}^{2 k}\left(\pi-\varphi_{j}(\eta)\right)\right) \\
& \leq 2\left(\sum_{j=1}^{2 k} \varphi_{j}(\eta) \wedge \frac{\pi}{2}\right) \wedge\left(\sum_{j=1}^{2 k}\left(\pi-\varphi_{j}(\eta)\right) \wedge \frac{\pi}{2}\right) \\
& =\pi \# \Lambda_{2 k}(\eta) \mathbf{1}_{\left\{\sum_{j=1}^{2 k} \varphi_{j} \leq k \pi\right\}}(\eta) \\
& \quad+2 \sum_{j \in \Lambda_{2 k}^{c}(\eta)} \varphi_{j}(\eta) \wedge\left(\pi-\varphi_{j}(\eta)\right) \mathbf{1}_{\left\{\sum_{j=1}^{2 k} \varphi_{j} \leq k \pi\right\}}(\eta)+\pi \# \Lambda_{2 k}^{c}(\eta) \mathbf{1}_{\left\{\sum_{j=1}^{2 k} \varphi_{j}>k \pi\right\}}(\eta)
\end{aligned}
$$




$$
\begin{aligned}
& \quad+2 \sum_{j \in \Lambda_{2 k}(\eta)} \varphi_{j}(\eta) \wedge\left(\pi-\varphi_{j}(\eta)\right) \mathbf{1}_{\left\{\sum_{j=1}^{2 k} \varphi_{j}>k \pi\right\}}{ }^{(\eta)} \\
& \leq 2\left(\sum_{j=1}^{2 k} \varphi_{j}(\eta)\right) \wedge\left(\sum_{j=1}^{2 k}\left(\pi-\varphi_{j}(\eta)\right)\right)
\end{aligned}
$$

where we wrote $\Lambda_{2 k}(\eta)=\left\{1 \leq j \leq 2 k: \varphi_{j}(\eta)>\frac{1}{2} \pi\right\}=\left\{1 \leq j \leq 2 k: \tau_{j} \eta>2\right\}$, and $\Lambda_{2 k}^{c}(\eta)=\left\{1 \leq j \leq 2 k: \varphi_{j}(\eta) \leq \frac{1}{2} \pi\right\}=\left\{1 \leq j \leq 2 k: \tau_{j} \eta \leq 2\right\}$. The symbol $\# \Lambda_{2 k}(\eta)$ denotes the number of elements in $\Lambda_{2 k}(\eta)$; sums over the empty set are to be interpreted as zero. The following proposition follows from (0.5) and the definition of $c_{2 k}(\eta)$.

PROPOSITION 0.2. The quantity $c_{2 k}(\eta)$ is uniformly bounded in $k \in \mathbb{N}$ and $\eta>0$ if and only if there exists a finite constant $C$ independent of $k$ and $\eta$ such that

1. $\# \Lambda_{2 k}(\eta) \leq C \sum_{j=1}^{2 k} \varphi_{j}(\eta) \wedge\left(\pi-\varphi_{j}(\eta)\right)$ for $\sum_{j=1}^{2 k} \varphi_{j}(\eta) \leq k \pi$, and

2. $\# \Lambda_{2 k}^{c}(\eta) \leq C \sum_{j=1}^{2 k} \varphi_{j}(\eta) \wedge\left(\pi-\varphi_{j}(\eta)\right)$ for $\sum_{j=1}^{2 k} \varphi_{j}(\eta)>k \pi$.

Notice that $\varphi_{j}(\eta) \wedge\left(\pi-\varphi_{j}(\eta)\right)=2 \arctan \left(\frac{\tau_{j} \eta}{2} \wedge \frac{2}{\tau_{j} \eta}\right)$ and that the expression $\varphi_{j}(\eta) \wedge\left(\pi-\varphi_{j}(\eta)\right)$ may be replaced by $\sin \varphi_{j}(\eta)=\frac{\tau_{j} \eta}{1+\frac{1}{4} \tau_{j}^{2} \eta^{2}}$ or by $\frac{\tau_{j} \eta}{2} \wedge \frac{2}{\tau_{j} \eta}$ without jeopardizing the conclusion in Proposition 0.2.

A result that looks nicer than Proposition 0.2 is available.

THEOREM 0.3. Let the notation be as in (the proof of) Theorem 0.1. Then, the following assertions are equivalent:

1. There exists a constant $C_{1}$ such that

$$
\#\left\{1 \leq j \leq 2 k: \varphi_{j}>\frac{\pi}{2}\right\} \wedge \#\left\{1 \leq j \leq 2 k: \varphi_{j} \leq \frac{\pi}{2}\right\} \leq C_{1} \sum_{j=1}^{2 k} \varphi_{j} \wedge\left(\pi-\varphi_{j}\right) .
$$

2. There exists a constant $C_{2}$ such that

$$
\left(\sum_{j=1}^{2 k} \varphi_{j}\right) \wedge\left(\sum_{j=1}^{2 k}\left(\pi-\varphi_{j}\right)\right) \leq C_{2} \sum_{j=1}^{2 k} \varphi_{j} \wedge\left(\pi-\varphi_{j}\right)
$$

In fact, if $C_{1}$ is such that (0.6) holds, then $C_{2}=\pi C_{1}+2$ satisfies (0.7). Conversely, if $C_{2}$ is a constant for which (0.7) holds, then (0.6) is true with $C_{1}=\frac{2 C_{2}}{\pi}$.

Proof. The result in Theorem 0.3 is a consequence of the following inequalities which are more or less self-explanatory and which looks pretty much like $(0.5)$ : 


$$
\begin{aligned}
& \frac{\pi}{2}\left(\#\left\{1 \leq j \leq 2 k: \varphi_{j}>\frac{\pi}{2}\right\} \wedge \#\left\{1 \leq j \leq 2 k: \varphi_{j} \leq \frac{\pi}{2}\right\}\right) \\
& \leq\left(\sum_{j=1}^{2 k} \varphi_{j}\right) \wedge\left(\sum_{j=1}^{2 k}\left(\pi-\varphi_{j}\right)\right) \leq 2\left(\sum_{j=1}^{2 k} \varphi_{j} \wedge \frac{\pi}{2}\right) \wedge\left(\sum_{j=1}^{2 k}\left(\pi-\varphi_{j}\right) \wedge \frac{\pi}{2}\right) \\
& =\left(\pi \#\left\{1 \leq j \leq 2 k: \varphi_{j}>\frac{\pi}{2}\right\}+2 \sum_{j=1, \varphi_{j} \leq \frac{1}{2} \pi}^{2 k} \varphi_{j}\right) \\
& \wedge\left(\pi \#\left\{1 \leq j \leq 2 k: \varphi_{j} \leq \frac{\pi}{2}\right\}+2 \sum_{j=1, \varphi_{j}>\frac{1}{2} \pi}^{2 k}\left(\pi-\varphi_{j}\right)\right) \\
& =\left(\pi \#\left\{1 \leq j \leq 2 k: \varphi_{j}>\frac{\pi}{2}\right\}+2 \sum_{j=1, \varphi_{j} \leq \frac{1}{2} \pi}^{2 k} \varphi_{j} \wedge\left(\pi-\varphi_{j}\right)\right) \\
& \wedge\left(\pi \#\left\{1 \leq j \leq 2 k: \varphi_{j} \leq \frac{\pi}{2}\right\}+2 \sum_{j=1, \varphi_{j}>\frac{1}{2} \pi}^{2 k} \varphi_{j} \wedge\left(\pi-\varphi_{j}\right)\right) \\
& \leq\left(\pi \#\left\{1 \leq j \leq 2 k: \varphi_{j}>\frac{\pi}{2}\right\}+2 \sum_{j=1}^{2 k} \varphi_{j} \wedge\left(\pi-\varphi_{j}\right)\right) \\
& \wedge\left(\pi \#\left\{1 \leq j \leq 2 k: \varphi_{j} \leq \frac{\pi}{2}\right\}+2 \sum_{j=1}^{2 k} \varphi_{j} \wedge\left(\pi-\varphi_{j}\right)\right) \\
& =\pi\left(\#\left\{1 \leq j \leq 2 k: \varphi_{j}>\frac{\pi}{2}\right\} \wedge \#\left\{1 \leq j \leq 2 k: \varphi_{j} \leq \frac{\pi}{2}\right\}\right) \\
& +2 \sum_{j=1}^{2 k} \varphi_{j} \wedge\left(\pi-\varphi_{j}\right)
\end{aligned}
$$

The conclusion in Theorem 0.3 follows from the inequalities in (0.8).

If $\varphi_{j}(\eta)=2 \arctan \left(\frac{1}{2} \tau_{j} \eta\right), 1 \leq j \leq 2 k$, then Theorem 0.3 can be rephrased as follows.

\section{THEOREM 0.4. The following assertions are equivalent:}

1. There exists a constant $C_{1}$ such that for all $\eta>0$ and $k \in \mathbb{N}$

$$
\#\left\{1 \leq j \leq 2 k: \tau_{j} \eta>2\right\} \wedge \#\left\{1 \leq j \leq 2 k: \tau_{j} \eta \leq 2\right\} \leq C_{1} \sum_{j=1}^{2 k} \frac{\tau_{j} \eta}{1+\frac{1}{4} \tau_{j}^{2} \eta^{2}}
$$


2. There exists a constant $C_{2}$ such that for all $\eta>0$ and for all $k \in \mathbb{N}$

$$
\left(\sum_{j=1}^{2 k} \varphi_{j}(\eta)\right) \wedge\left(\sum_{j=1}^{2 k}\left(\pi-\varphi_{j}(\eta)\right)\right) \leq C_{2} \sum_{j=1}^{2 k} \frac{\tau_{j} \eta}{1+\frac{1}{4} \tau_{j}^{2} \eta^{2}}
$$

COROLLARY 0.5. Let the sequence of positive real numbers $\left\{\tau_{j}: j \in \mathbb{N}\right\}$ satisfy (0.9) in Theorem 0.4, and let $A$ be the generator of a bounded analytic semigroup in a Banach space $(X,\|\cdot\|)$ with domain $D(A)$. Then, the Crank-Nicolson iteration scheme

$$
\left(I-\frac{1}{2} \tau_{n+1} A\right) x_{n+1}=\left(I+\frac{1}{2} \tau_{n+1} A\right) x_{n}, x_{0} \in D(A),
$$

is stable in the sense that $\sup _{n \in \mathbb{N}}\left\|x_{n}\right\|<\infty$.

Proof. Corollary 0.5 is a consequence of Theorem 0.4 in conjunction with Theorem 0.1 .

It is conjectured that a sequence $\left\{\tau_{j}: j \in \mathbb{N}\right\}$ that together with its inverse $\left\{1 / \tau_{j}: j \in \mathbb{N}\right\}$ is polynomially bounded satisfies (0.9), and hence that for such a sequence, the corresponding Crank-Nicolson scheme $(0.11)$ is stable provided that the operator $A$ generates a bounded analytic semigroup.

An argument in favor of this conjecture reads as follows. Suppose that there exists a strictly increasing continuous function $p:(0, \infty) \rightarrow(0, \infty)$ which is surjective and a function $q:(0, \infty) \rightarrow(0, \infty)$ which is strictly positive on $[0,1]$ such that the limit, pointwise in $(s, \eta) \in(0, \infty) \times(0, \infty)$,

$$
\lim _{k \rightarrow \infty} \frac{p(\lceil 2 k s \eta\rceil)}{p(2 k \eta)}=q(s), \text { uniformly in } \frac{1}{2} \leq \eta \leq 1 \text { for } s>0 .
$$

Here, $\lceil\eta\rceil$ denotes the integer $\ell$ satisfying $\ell-1<\eta \leq \ell$, and $\lfloor\eta\rfloor$ is the integer $\ell$ satisfying $\ell \leq \eta<\ell+1$. Upon defining $\xi>0$ by the equality $\eta p\left(2 k \xi^{-1}\right)=2$, it follows that $(0.9)$ is equivalent to

$$
\frac{\xi}{2 k}\left\{\left(2 k-\left\lfloor\frac{2 k}{\xi}\right\rfloor\right) \wedge\left\lfloor\frac{2 k}{\xi}\right\rfloor\right\} \leq C_{1} \int_{0}^{\xi} \frac{2 p\left(\left\lceil 2 k s \xi^{-1}\right\rceil\right)}{p\left(2 k \xi^{-1}\right)} \frac{1}{1+\left(\frac{p\left(\left\lceil 2 k s \xi^{-1}\right\rceil\right)}{p\left(2 k \xi^{-1}\right)}\right)^{2}} d s,
$$

for $1 \leq \xi \leq 2 k$. Upon letting $k \rightarrow \infty$ in (0.13), it follows that

$$
(\xi-1) \wedge 1 \leq C_{1} \int_{0}^{\xi} \frac{2 q(s)}{1+q(s)^{2}} d s, \quad \xi \geq 1 .
$$

However, an inequality of the form (0.14) holds automatically. In view of (0.12), the arguments leading to (0.14) can be reversed to obtain (0.9), and hence, the stability of the Crank-Nicolson scheme (0.11) with step size $\tau_{j}=p(j)$, where $\eta \mapsto p(\eta)$ is 
an increasing polynomial, provided that the operator $A$ generates a bounded analytic semigroup. Moreover, it is easily seen that a sequence of strictly positive numbers $\left\{\tau_{j}: j \in \mathbb{N}\right\}$ satisfies (0.9) if and only if the sequence of its reciprocals does so. In addition, it is not so difficult to prove that a sequence $\left\{\tau_{j}: j \in \mathbb{N}\right\}$ satisfies (0.9) if and only if there exist positive integers $m$ and $n$ and a finite constant $C_{m, n}$ such that the following inequality holds:

$$
\begin{aligned}
& \#\left\{1 \leq j \leq 2 k: \tau_{j} \eta>2^{m}\right\} \wedge \#\left\{1 \leq j \leq 2 k: \tau_{j} \eta \leq 2^{-n}\right\} \\
& \quad \leq C_{m, n} \sum_{j=1}^{2 k} \frac{\tau_{j} \eta}{1+\frac{1}{4} \tau_{j}^{2} \eta^{2}} .
\end{aligned}
$$

Let $\left\{\tau_{j}: j \in \mathbb{N}\right\}$ be a sequence of positive real numbers for which there exists an increasing polynomial $\eta \mapsto p(\eta)$ and constants $\gamma_{1}>0, \gamma_{2}<\infty$, such that $\gamma_{1} p(j) \leq$ $\tau_{j} \leq \gamma_{2} p(j), j \in \mathbb{N}$. Then, the previous arguments together with (0.15) show that such a sequence satisfies (0.9), so that the Crank-Nicolson scheme with step sizes $\tau_{j}, j \in \mathbb{N}$, is stable.

\title{
REFERENCE
}

[1] Jan A. van Casteren, On the Crank-Nicolson scheme once again, J. Evol. Equ. (2011), doi:10.1007/ s00028-010-0084-9

\author{
J. A. van Casteren \\ Department of Mathematics and Computer Science, \\ University of Antwerp, \\ Middelheimlaan 1, 2020 Antwerp, \\ Belgium \\ E-mail: jan.vancasteren@ua.ac.be
}

